\title{
BTDAzo - a photoswitchable TRPC5 channel activator
}

\author{
Markus Müller, ${ }^{a}$ Konstantin Niemeyer, ${ }^{b}$ Nicole Urban, ${ }^{b}$ Navin K. Ojha, ${ }^{c}$ Frank Zufall, ${ }^{c}$ Trese Leinders- \\ Zufall, ${ }^{\mathrm{c}}$ Michael Schaefer, ${ }^{\mathrm{b}}$ Oliver Thorn-Seshold ${ }^{\mathrm{a} *}$
}

\footnotetext{
[a] M. Müller, Dr. O. Thorn-Seshold

Department of Pharmacy

LMU Munich

Butenandtstrasse 7, Munich 81377, Germany

E-mail: oliver.thorn-seshold@cup.Imu.de

[b] K. Niemeyer, N. Urban, Prof. Dr. M. Schaefer

Rudolf-Boehm-Institute of Pharmacology and Toxicology

Leipzig University

Härtelstraße 16-18, 04107 Leipzig, Germany

[c] Dr. N.K. Ojha, Prof. Dr. F. Zufall, Prof. Dr. T. Leinders-Zufall

Center for Integrative Physiology and Molecular Medicine

Saarland University

Kirrbergerstraße 100, 66421 Homburg, Germany
}

\begin{abstract}
Photoswitchable reagents to modulate protein activity are powerful tools for high-spatiotemporal-precision control over endogenous biological functions. TRPC5 is a $\mathrm{Ca}^{2+}$-permeable cation channel with distinct tissue-specific roles, ranging from synaptic function to hormone regulation. Achieving spatially-resolved control over TRPC5 activity in particular cells or tissues, and temporal regulation in targeted cells, are therefore crucial milestones towards understanding and harnessing the biology of TRPC5. Here we develop the first photoswitchable TRPC5-modulating reagent, BTDAzo, towards reaching this goal. BTDAzo can photocontrol TRPC5 currents in cell culture, as well as controlling endogenous TRPC5-based neuronal $\mathrm{Ca}^{2+}$ responses in mouse brain slices. BTDAzos are also the first reported azo-benzothiadiazines, an accessible and conveniently derivatised azoheteroarene that features excellent two-colour photoswitching. BTDAzo's TRPC5 control across relevant channel biology settings makes it appropriate for a range of dynamically reversible photoswitching studies in TRP channel biology, aiming to decipher the various biological roles of this centrally important ion channel.
\end{abstract}

\section{Introduction}

The twenty-eight Transient Receptor Potential (TRP) channels have crucial roles in sensing and integrating a wide range of stimuli. ${ }^{[1]}$ Better-known members include TRPV1 (heat) and TRPM8 (cold) for which a 2021 Nobel Prize was awarded, and TRPA1 (electrophiles). As these channels are expressed in many tissues but play different biological roles in these tissues, ${ }^{[2]}$ potentially with timedependent aspects, a range of photoswitchable ligands have been actively developed to elucidate their tissue- and time-specific roles through spatiotemporally precise modulation. ${ }^{[3]}$ Notable photoswitchable TRP ligands include analogues of diacylglycerols (PhoDAGs) ${ }^{[4,5]}$ and of small polar GSK ligands ${ }^{[6]}$ for TRPC2,3,6; azo-vanilloids (azCA4 ${ }^{[7]}$, red-azCA4 ${ }^{[8]}$ ) for TRPV1; and TRPswitch ${ }^{[9]}$ for TRPA1.

TRPC5 is implicated in a range of tissue-dependent roles in physiology as well as in disease, with brain functions from synaptic plasticity and hormone regulation to potential importance in metabolic medicine ${ }^{[2,10-12]}$. TRPC1,4 and 5 share substantial structural overlap which drives the typically poor channel selectivity of TRPC1/4/5 ligands; however, drug discovery for TRPC5 has recently yielded a treasure trove of valuable ligands. ${ }^{[13,14]}$ Weak antagonists were first identified from screening, ${ }^{[15]}$ before it was identified by Waldmann as a target of the potent but nonselective TRPC4/5-targeting natural product agonist Englerin $A^{[16,17]}$ (Fig. 1a). Moderately potent synthetic agonist BTD was also identified by Schaefer, with the unusual feature of excellent selectivity for TRPC5 and no activity on TRPC4. ${ }^{[18]}$ Boehringer-Hydra disclosed highpotency xanthine antagonist leads intended to treat anxiety, ${ }^{[19,20]}$ that Bon and coworkers characterised as effective TRPC5 modulator tool compounds ${ }^{[21]}$; Bon developed these xanthines further to reach intriguing selectivity and mixed agonist-antagonist profiles (AM237; Fig 1a) ${ }^{[22]}$ as well as identify the Pico145 binding site ${ }^{[23,24]}$.<smiles>Cc1ccc2c(c1)NC(CCC(=O)NCCCOC13CC4CC(CC(C4)C1)C3)=NS2(=O)=O</smiles>

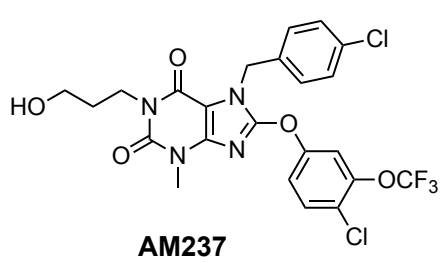<smiles>CC(C)[C@@]12C[C@H](OC(=O)CO)[C@@](C)(O1)[C@H]1CC[C@@H](C)[C@H]1[C@H]2OC(=O)/C=C/c1ccccc1</smiles>

b<smiles>COc1cc(N=Nc2ccc3c(c2)S(=O)(=O)N=C(CCC(=O)NCCCOC24CC5CC(CC(C5)C2)C4)N3)cc(OC)c1OC</smiles>

Figure 1. Design. (a) Known TRPC5 agonists BTD, Englerin A, AM237. (b) BTDAzo, the best photoswitchable agonist of the series btda1-7 (cf. Fig S1).

However, no photoswitchable TRPC5 ligands have yet been reported, which could help to study its tissue- and time-resolved biological roles. We therefore wished to 
create TRPC5-selective photoswitchable ligands, with minimal effects on TRPC1/4 channels, as high spatiotemporal-precision tools with useful biochemical selectivity. BTD represented a good basis structure for this goal. Although its potency is moderate $\left(\mathrm{EC}_{50} \sim 1.4 \mu \mathrm{M}\right)$, it only activates homomeric TRPC5 or heteromeric TRPC[1/4/]5 channel complexes that contain TRPC5 subunits, making it a more selective tool than e.g. AM237 or Englerin A. We set out to create a photoswitchable analogue of BTD for applications in researching the role of endogenous TRPC5 in mammalian cells and tissue slices.

\section{Results and Discussion}

\section{Design and Synthesis}

The TRPC5 binding mode of BTD is unknown, and structure-activity-relationship data are limited (the 4-methyl group on the benzothiadiazine can be deleted, but shorter spacers between the adamantane and benzothiadiazine abolish activity). ${ }^{[18]}$ We tested applying the bidirectional photoswitch azobenzene to the BTD scaffold at each end, presuming that one end could be sterically tolerated.

Firstly, we extended the azobenzene from the benzothiadiazine ring, though in para to the nitrogen. This was chosen hoping that this unknown azoheteroarene that is electronically similar to a para-anilide, could provide similarly desirable photoswitching properties, as are necessary for creating an effective photopharmaceutical: i.e. bidirectional photoswitching with near-UV and bluegreen light each achieving high photostationary states (PSSs). ${ }^{[25]}$ We varied the azophenyl substitution pattern to scan for different polarities, PSSs, and $Z \rightarrow E$ spontaneous relaxation rates: comparing unsubstituted, with paramethoxy and trimethoxy (improved PSSs), and parahydroxy (faster relaxation) designs btda1-4 (Fig S1). Secondly, we replaced the hydrophobic adamantyl group with the azobenzene: retaining the oxyether attachment site because of its beneficial effects on photoswitching, ${ }^{[26]}$ and employing an unsubstituted, para-methoxy, or a bulky adamantyl-mimicking bis-isopropyl azobenzene, in designs btda5-7 (Fig S1).

The seven candidates btda1-7 were assembled over three to six least linear steps, with good yields in all steps for except the final O-methylations of btda2-3; representative synthesis of btda3 is shown in Scheme 1. btda1-7 were typically obtained in $10 \mathrm{mg}$ batches and excellent purity, sufficient for biological evaluations (see Supporting Information for details).

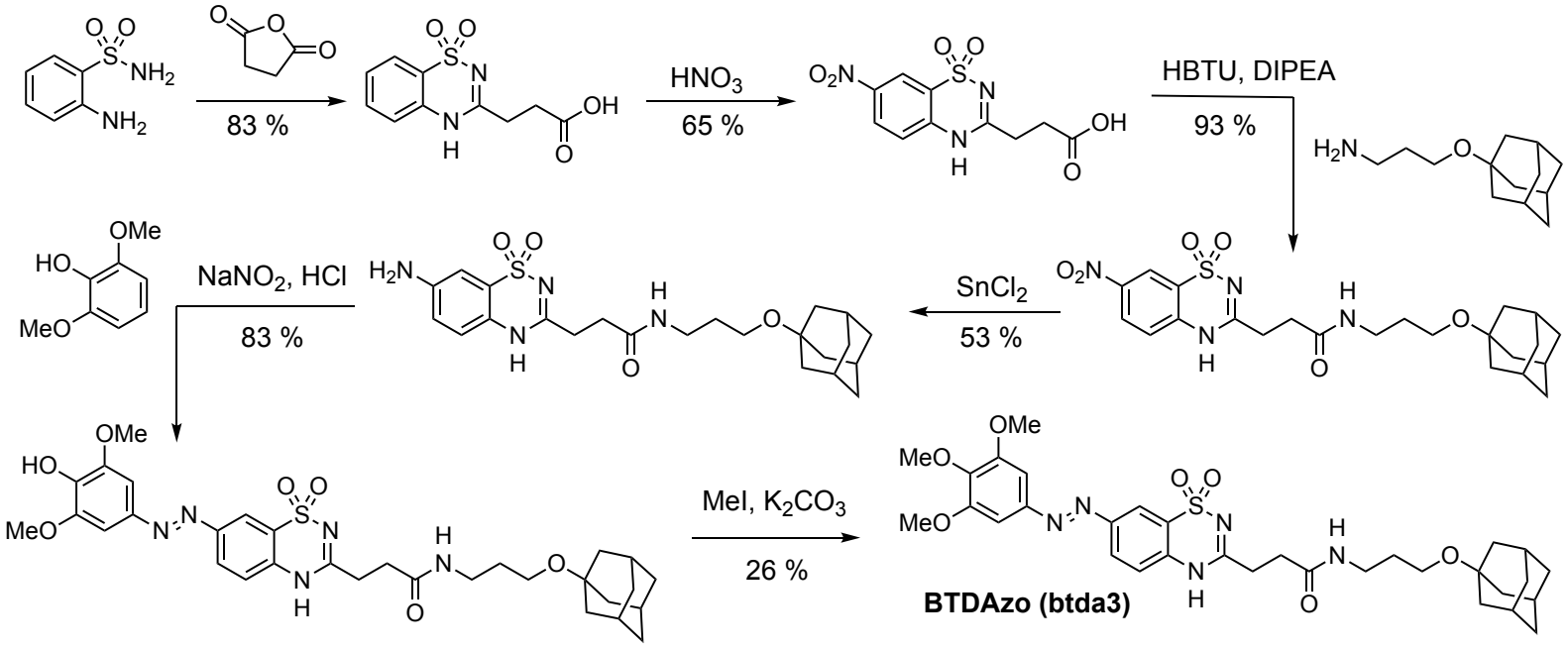

Scheme 1. Synthesis of BTDAzo (btda3).

\section{Photoswitchability}

Pleasingly, the as-yet unstudied phenylazobenzothiadiazine (btda1-4) proved to be an excellent photoswitch, with performance recalling that of paraanilides. As expected for mono/bis-para-alkoxy azobenzenes, ${ }^{[26]}$ btda5-7 also had high PSS ratios and efficient photoswitching. As btda3 later gave the best biological performance, it was renamed BTDAzo, and studied in detail. Like classical azobenzenes, the $E$-isomer is thermodynamically preferred, and all- $E$ populations are reached by maintaining DMSO solution stocks at $60^{\circ} \mathrm{C}$ overnight. BTDAzo could be reversibly photoswitched between majority- $E$ and majority- $Z$ populations, reaching solvent-dependent PSSs with up to $94 \% Z(380 \mathrm{~nm})$ and $87 \% E(520 \mathrm{~nm})$ in apolar solvents that mimic the expected cellular lipid environment for this hydrophobic compound
(Fig 2a-c; Fig S2). The spectral separation between $E$ and $Z$ bands that results in the $Z$ absorption minimum being located at the same wavelength as the $E$ absorption maximum (Fig 2d) is the driver of the high-Z PSS.

Photoswitching in aqueous media was also excellently complete and was photoreversible over many cycles with no photoinstability noted (Fig 2e). $Z \rightarrow E$ thermal relaxation below $40^{\circ} \mathrm{C}$ was significantly slower than the timescales of channel switching experiments (Fig S3). PSS spectra and discussions of photochemistry for other btda derivatives are given in the Supporting Information at Fig S3-4. 


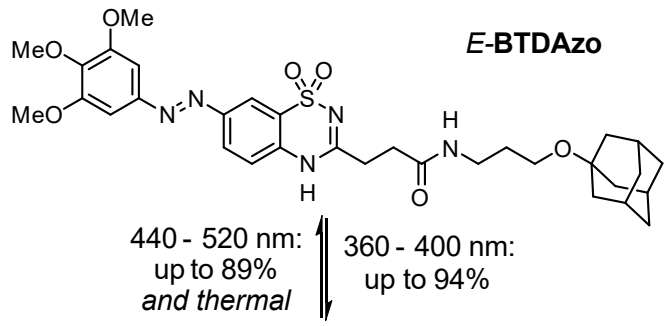<smiles>[Z10][R16]=O</smiles>

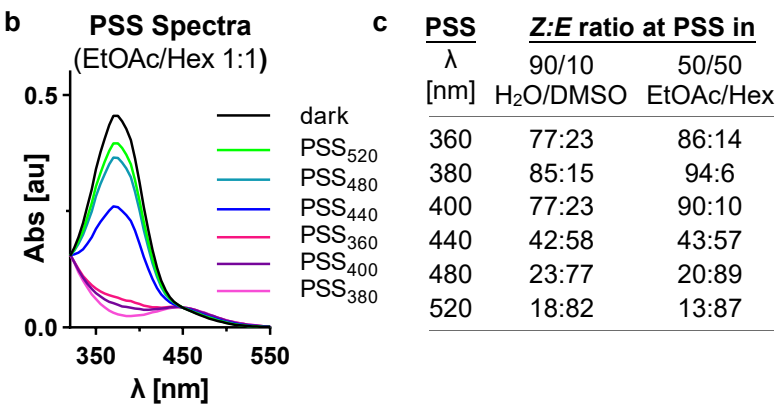

d Isolated Spectra

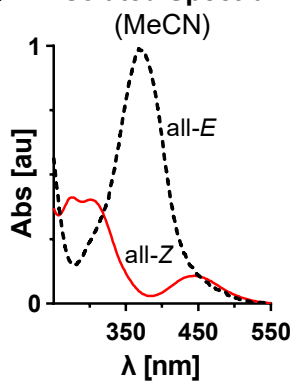

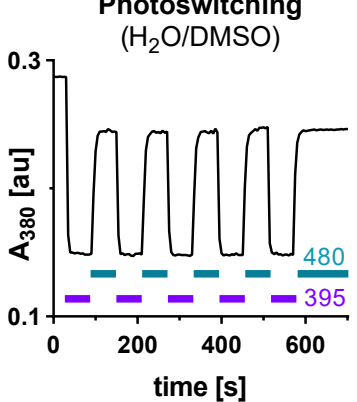

Figure 2. Photocharacterisation. (a) $E \rightarrow Z$ isomerisations of BTDAzo. (b) $Z: E$ ratios at PSS depending on environment. (c) PSS spectra of BTDAzo in $\mathrm{H}_{2} \mathrm{O} /$ DMSO. (d) Spectra of pure E- and Z-BTDAzo (inline HPLC detection). (e) BTDAzo can be reversibly photoswitched between PSS states.

\section{Cellular TRPC5 Photopharmacology}

We initially screened btda1-7 as photoswitchable TRPC5 agonists in cells, using human embryonic kidney cell line HEK293 stably transfected to express a mouse

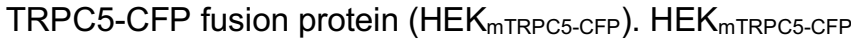
cell suspensions were loaded with the fluorescent $\mathrm{Ca}^{2+}$ indicator dye precursor Fluo-4/AM, washed, and dispensed into black pigmented clear-bottom 384-well microplates to monitor ion channel opening in a custommade fluorescence imaging plate reader device. By adding serially diluted compounds to single wells, this is a convenient high-throughput method to obtain concentration-response curves before and during controlled application of ultraviolet light to the bottom of the microplate. We wished to use alternating cycles of moderate intensity $360 / 447 \mathrm{~nm}$ light for substantial $E \rightleftarrows Z$ photoswitching. The fluorescent $\mathrm{Ca}^{2+}$ indicator Fluo-4 was

either excited at $447 \mathrm{~nm}$ for combined detection and offswitching, or at low intensities of 470-nm light to achieve a lower impact on btda photostationary states due to the small extinction coefficients in the cyan (Fig S4).

BTDAzo (bdta3) was the best hit from this screening: it gave highly repeatable, bidirectional $\mathrm{Ca}^{2+}$ influx signals with low basal activity as the relaxed $E$ isomer and robust responses upon photoswitching with $365 \mathrm{~nm}$ light (Fig 3a). BTDAzo showed several outstanding features. Firstly, the potency of Z-BTDAzo (EC $\mathrm{E}_{50} 1.5 \mu \mathrm{M}$, Fig $\mathbf{3 b}$ ) was as good as that of its parent molecule BTD $\left(E_{50} 1.4 \mu \mathrm{M}\right)^{[18]}$; potency matching is only very rarely achieved in photopharmacology. ${ }^{[27]}$ Secondly, not only was all-EBTDAzo at $50 \mu \mathrm{M}$ fully inactive on TRPC5, and nearly nonresponsive to $470 \mathrm{~nm}$ imaging (initial $60 \mathrm{~s}$ of Fig 3a), but photoswitching of $50 \mu \mathrm{M}$ mostly-Z-BTDAzo with $440 \mathrm{~nm}$ reduced channel currents to below those seen with mostly$Z$ BTDAzo at just $1.5 \mu \mathrm{M}$. It is BTDAzo's combination of activity exclusively in the Z-isomer, with remarkably efficient $Z \rightarrow E$ photoswitching at $440 \mathrm{~nm}$ in cells, that gives it such effective bidirectional photoswitching of bioactivity. Thirdly, no "biological fatigue" was seen, as photoswitching of was highly repeatable over many cycles. Thus BTDAzo could be a robust tool for complex studies (particularly since, as a lit-active photoswitch, it avoids background bioactivity in non-illuminated cells). The other btdas were not good tool compounds: btda1,2,4 gave much slower bulk photoresponses, while btda5-7 were inactive (see Fig S5 and Supporting Information).

Since the plate imaging device uses single-color light-emitting diodes for Fluo-4 detection as well as for BTDAzo photoswitching, no continuous spectral information could be gathered in this assay. We therefore used single-cell $\mathrm{Ca}^{2+}$ imaging with a Xenon lamp-equipped monochromator as excitation light source to study the cellular action spectra (channel current dependency on wavelength) and cellular power spectra (channel current dependency on applied photon flux), and to optimise assay illumination protocols. $E \rightarrow Z$ action spectra showed optimal photo-switch-on response to ca. 360-370 nm (Fig 3c-d) when measured in flux-limited conditions designed to minimise photobleaching (power spectrum Fig S6a-b). $Z \rightarrow E$ action spectra showed optimal photo-switch-off response to ca. $420-460 \mathrm{~nm}$ (Fig 3e-f) in flux-limited conditions (power spectrum Fig S6c-d). The striking action spectrum peak sharpness (Fig 3d), and the mismatch between the poor photoswitching completion in homogeneous polar media such as DMSO:water (Fig 1c) and the excellent performance in cells, indicate that cellfree measurements are only approximately predictive of the cellularly-relevant photoswitching of this lipophilic photoswitch (discussions in Supporting Information at Fig S4 [relaxation timescales] and Fig S6 [action spectra]). The power spectra additionally highlight that bulk $E \rightarrow Z$ and $Z \rightarrow E$ photoswitching in the cellular setting proceed at comparable rates $\left(50 \%\right.$ conversion at $30 \mathrm{~mJ} / \mathrm{cm}^{2}$ for $360 \mathrm{~nm}$ and $50 \mathrm{~mJ} / \mathrm{cm}^{2}$ for $440 \mathrm{~nm}$; Fig S6); this is a reminder that the ratio of $E / Z$ extinction coefficients is more important for photoswitching performance in microscopy, than are their absolute magnitudes (ca. tenfold lower at $440 \mathrm{~nm}$ than at $360 \mathrm{~nm}$ ). 
HEK cell $\mathrm{Ca}^{2+}$ influx photomodulation with BTDAzo (365/447 nm illumination cycles) - plate imager

a

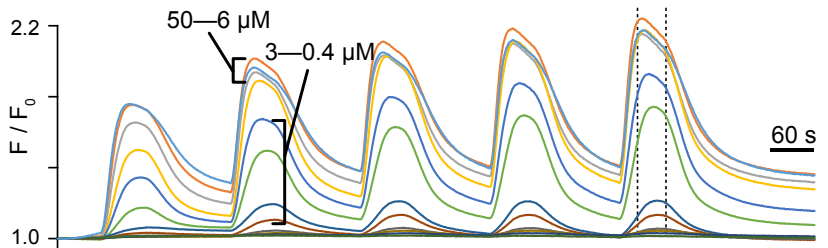



HEK cell $\mathrm{Ca}^{2+}$ influx photomodulation with BTDAzo - single cell fluorescence microscopy analysis

C
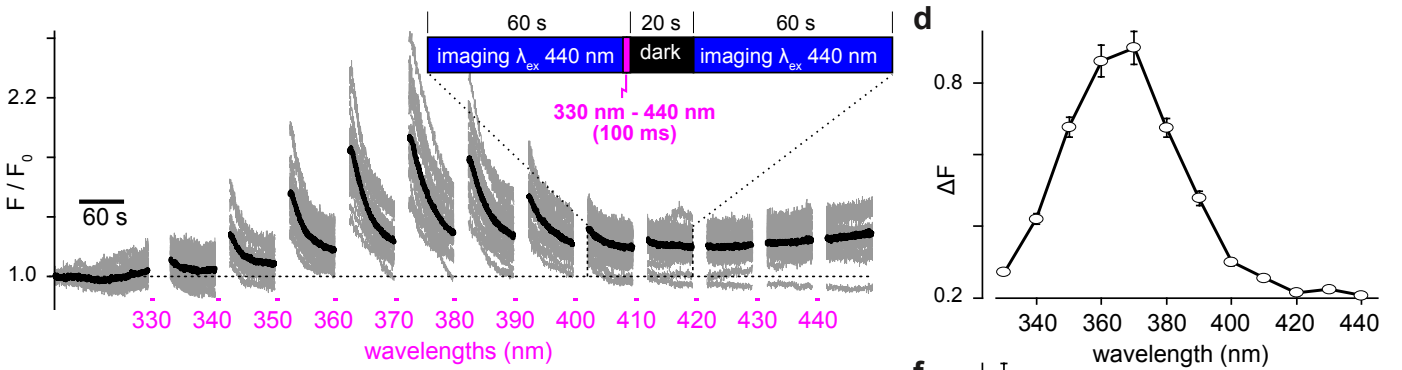

e
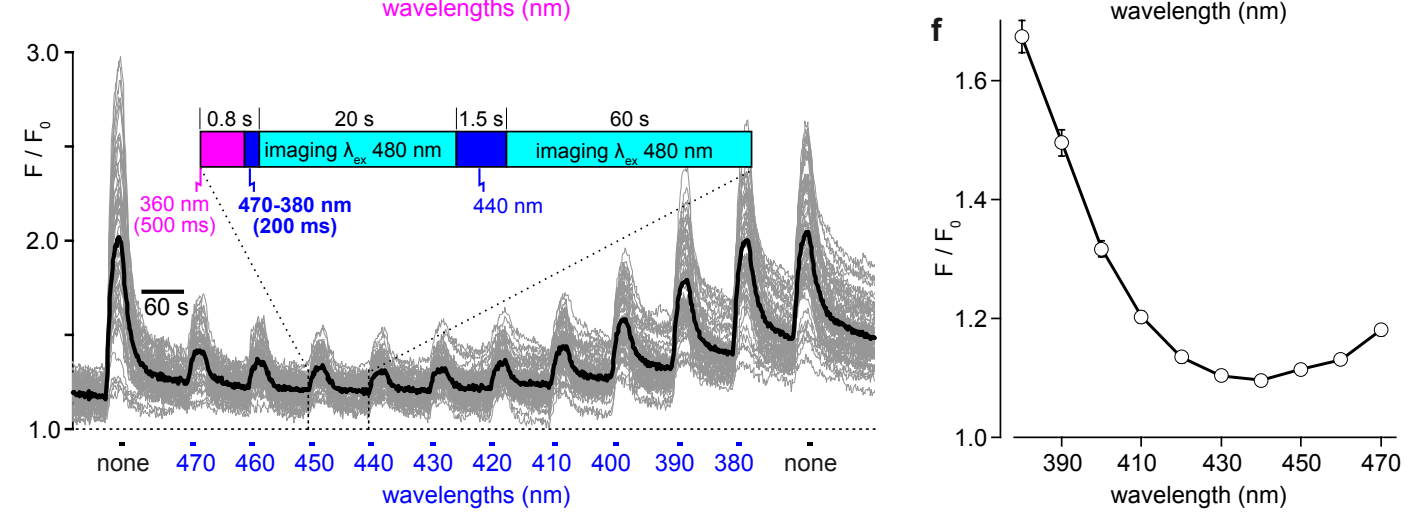

g

Whole-cell recordings - BTDAzo-photomodulated electrophysiology

$440 \mathrm{~nm}=$

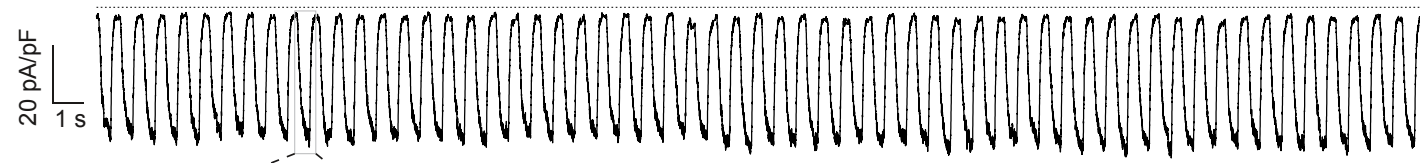

$\mathbf{h}$



i $\quad$ TRPC5



j TRPC1:TRPC5


Figure 3: Cellular TRPC5 photoswitching with BTDAzo. (a-b) Reversible $\mathrm{Ca}^{2+}$ influx modulation with BTDAzo under cycles of $365 / 447 \mathrm{~nm}$ illumination,

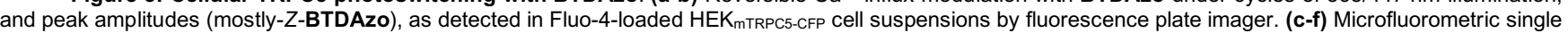
cell analysis (60 grey traces: single cells; black trace: averaged signal) of TRPC5 activation measured with a monochromator-equipped Xenon light source. (c-d) Cellular action spectrum for stimulation of $\mathrm{Ca}^{2+}$ influx into single HEK $\mathrm{Ca}^{2+}$ influx (after $E \rightarrow Z$ isomerisation) by $Z \rightarrow E$ BTDAzo isomerisation at various wavelengths. (g-h) Electrophysiological whole-cell recordings of TRPC5 currents in voltage clamp $\left(V_{\mathrm{h}}=-80 \mathrm{mV}\right)$ mode. (g) lonic currents in a TRPC5-expressing HEK293 cell during 60 consecutive cycles of $360 / 440 \mathrm{~nm}$ illumination in the presence of $10 \mu \mathrm{M}$ BTDAzo. Dotted line: zero current level. (h) Magnification of a single on-off cycle taken from the trace shown in (g). (i) I/V curves of whole cell currents in a voltage-clamped TRPC5-expressing cell exposed to $360 \mathrm{~nm}$ and $440 \mathrm{~nm}$ light with $10 \mu \mathrm{M}$ BTDazo in the bath solution. (j) Patch-clamp traces obtained as in (i), but from a TRPC1/TRPC5-co-expressing cell. Note the distinct shape of the I/V curve with smaller inward current component.

We next performed patch clamp experiments, a nonoptical current readout that can orthogonally confirm and characterise the specificity of channel modulation. In the presence of $10 \mu \mathrm{M}$ BTDAzo, on/off-photoswitching of ionic currents was fully reproducible over 60 cycles (Fig $\mathbf{3 g}$ ) with $360 \mathrm{~nm}$ rapidly opening then $440 \mathrm{~nm}$ almost fully reclosing 
channels to baseline conductivity (Fig $3 \mathbf{h}$ ). The efficient channel closure following $440 \mathrm{~nm}$ illumination was seen over the entire current-voltage plot range (Fig 3i).

BTD is a strong tool compound because of its high selectivity for TRPC5-containing channels, ${ }^{[18]}$ while most other TRPC5 ligands also target TRPC4. To test whether BTDAzo retains this TRPC5-selectivity, we imaged TRPC4-expressing HEK293 cells in FLIPR, and pleasingly saw no TRPC4-mediated photostimulation of $\mathrm{Ca}^{2+}$ influx responses under any of the BTD derivatives applied at concentrations up to $50 \mu \mathrm{M}$, including BTDAzo (Fig S7).
Patch clamp recordings in TRPC1/TRPC5 co-expressing cells exposed to $10 \mu \mathrm{M}$ BTDAzo, however, showed a strong photocontrol of inward and outward currents with a characteristic shape of the I/V curve known to result from the assembly of TRPC1 and TRPC5 channel subunits into heteromeric channel complexes (Fig $3 \mathbf{j}$ ). This finding is reminiscent of the parent compound BTD, which also activates heteromeric TRPC1/C5 channel complexes.

Taken together, we conclude that BTDAzo is a selective, fully reversible "binary performance" photoswitchable agonist for cellular TRPC5. a





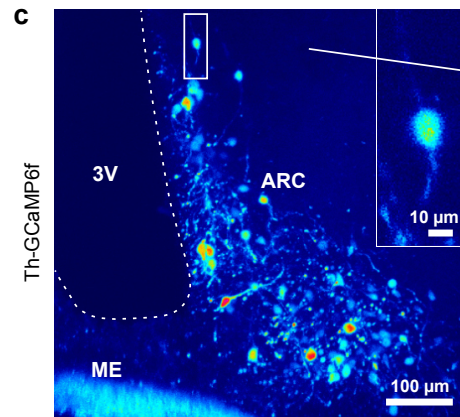

low $\mathrm{Ca}^{2+}$ high $\mathrm{Ca}^{2+}$

d
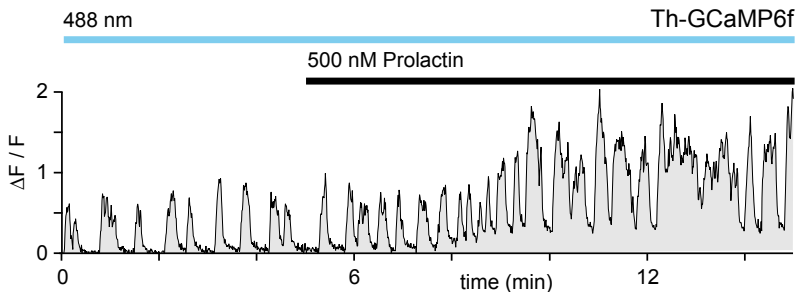

e $488 \mathrm{~nm}$ Th-GCaMP6f

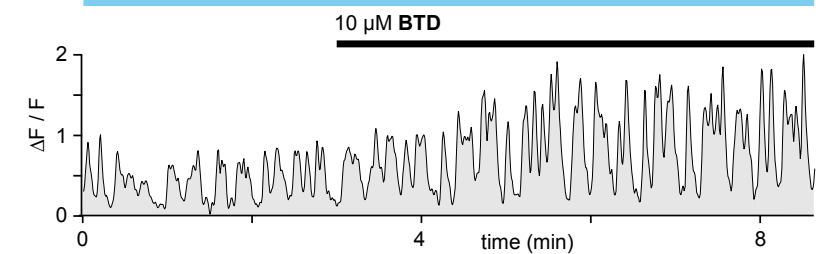

$\mathbf{f}$
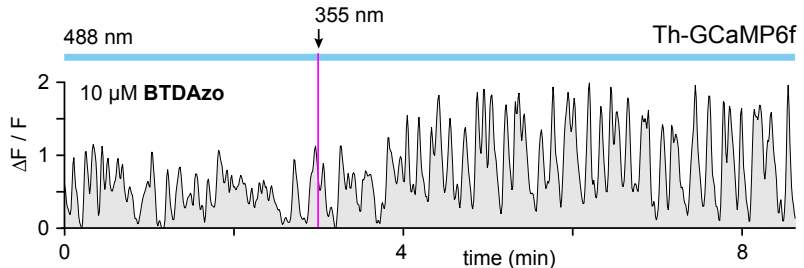

g
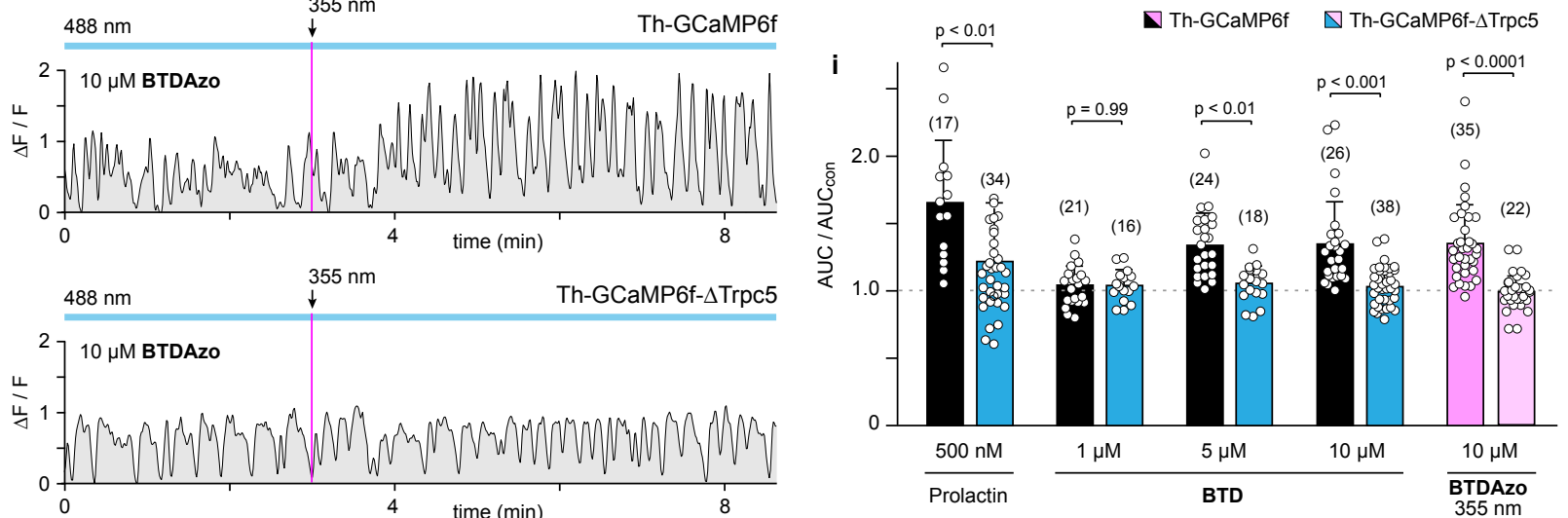

Figure 4: BTDAzo photocontrols endogenous TRPC5-dependent $\mathrm{Ca}^{2+}$ responses in mouse brain. (a) Cascade model for TRPC5-dependent cation channel activation in Th+ neurons of the hypothalamic arcuate nucleus (ARC) after stimulation of the prolactin receptor (PrIR). (b) Cartoon of the coronal brain slice, and region of the ARC (blue). Dashed box indicates the ARC region in (c). (c) Pseudocoloured image of Th+ neurons expressing GCaMP6f in a mouse brain slice. Inset: Zoom on a GCaMP6f neuron from the dorsomedial region of the ARC. (3V, third ventricle; ME, median eminence). (d-g) Original traces of spontaneous Ca ${ }^{2+}$ responses in Th+ neurons of Th-GCaMP6f mice, stimulated with prolactin (d), BTD (e), or BTDAzo followed by $E \rightarrow Z$ isomerisation using a $355 \mathrm{~nm}$ UV laser pulse (f-g). Panel (g) shows data for the TRPC5-deficient Th+ neuron of Th-GCaMP6f- $\triangle$ Trpc5 mouse. (h) Area under the curve (AUC) of Ca ${ }^{2+}$ signals when applying BTDAzo under 488 or $355 \mathrm{~nm}$ in wildtype or Trpc5 deficient Th+ neurons, compared with wildtype cosolvent only (Con) or BTD controls. Two-way ANOVA: F(3, $158)=3.011, p<0.05$. (i) Wildtype or knockout AUCs normalized to their controls (cosolvent or 488nm stimulation of BTDAzo) show prolactin, BTD, and BTDAzo after $355 \mathrm{~nm}$ photoswitching stimulate TRPC5-dependent $\mathrm{Ca}^{2+}$ increases. Kruskal-Wallis ANOVA: $X^{2}(9)=95.24, p<0.00001$. [(h-i): number of cells is indicated in parentheses above each bar; for full description of material methods and statistics see Supporting Information.] 


\section{Tissue slice photopharmacology - hypothalamic mouse dopamine neurons}

We now wished to use BTDAzo to probe the role of TRPC5-dependent responses in dopamine (i.e. tyrosine hydroxylase-positive, $\mathrm{Th}+$ ) neurons of the hypothalamic arcuate nucleus (ARC) (Fig 4a). TRPC5 contributes to both spontaneous oscillatory activity and persistent activation after stimulation with maternal signalling hormone prolactin, which is essential for normal prolactin homeostasis of the body (Fig 4a). ${ }^{[1]}$

We took slices through the ARC of mice expressing the $\mathrm{Ca}^{2+}$ indicator GCaMP6f in Th+ neurons (Fig 4b-c) to test whether BTDAzo illumination could achieve photocontrol over $\mathrm{Ca}^{2+}$ responses at endogenous TRPC5 expression levels. Spontaneous $\mathrm{Ca}^{2+}$ responses in $\mathrm{Th}+$ neurons after treatment with BTDAzo and $355 \mathrm{~nm}$ photoactivation matched those upon treatment with either prolactin or BTD (Fig 4d-f). Genetic deletion of the TRPC5 channel in the Th-GCaMP6f- $\Delta$ Trpc5 mouse prevented the increase in $\mathrm{Ca}^{2+}$ activity in Th+ neurons (Fig $\mathbf{4 g}$ ), indicating the channel selectivity of BTDAzo in the slice setting. The area under the curve (AUC) of the $\mathrm{Ca}^{2+}$ signals quantifies that BTDAzo does not induce changes under $488 \mathrm{~nm}$ illumination alone, but needs both isomerisation with 355 $\mathrm{nm}$ light and TRPC5 expression to generate $\mathrm{Ca}^{2+}$ rises ( $\mathrm{p}$ $<0.0001$ ) (Fig 4h-i).

Thus, BTDAzo can be used in complex tissue slice settings as a highly selective direct activator of the TRPC5 channel complex that acts only upon $E \rightarrow Z$ photoswitching to measurably increase sustained influxes of $\mathrm{Ca}^{2+}$ mimicking those seen with the endogenous activator, prolactin.

\section{Conclusion}

Photopharmaceuticals are proving to be powerful and flexible tools to non-invasively manipulate and study biological processes, with high temporal and spatial resolution that is appropriate to their function. ${ }^{[28,29]}$

We here report the first photoswitchable ligand for the ion channel TRPC5. BTDAzo can reversibly photoswitch cellular channel activity between opened (conducting) and full baseline states, and its potency and selectivity are additional promising features for future research. Its channel activation is potent enough to be applicable not only in overexpression systems, but in brain tissues with endogenous TRPC5 expression levels, and its pharmaceutical properties make it suitable for tissue slice use. Following the proof of concept brain slice assay we have tested, we consider BTDAzo as a valuable tool to investigate the various roles of TRPC5 (and TRPC4) in brain function, including their potential in the study and treatment of metabolic disease. ${ }^{[12]}$

We believe this is also the first report of azobenzothiadiazines as photoswitches for cell biology. It can give favourably complete bidirectional photoswitching with rapid response under biologically available wavelengths, and its easy synthesis and handling recommend it for use towards other photopharmaceuticals.

Challenges for the ongoing development and use of TRPC5 photopharmaceuticals in focus in our group include (i) improving ligand potency, and (ii) improving solubility and bioavailability, which should broaden the scope of applications of these ligands towards real-time in vivo uses. ${ }^{[30]}$ Nevertheless, BTDAzo is a reliable, potent TRPC5 photoswitch that can easily find applications in cell culture and slice use towards a better understanding of this truly remarkable protein.

\section{Acknowledgements}

We thank the German Research Foundation (DFG: SFB TRR 152 number 239283807 projects $\mathrm{P} 10$ to FZ/TLZ, P18 to MS, P24 to OTS; Emmy Noether number 400324123 to OTS) for funding.

Keywords: TRPC5; photopharmacology; optical control; photoswitch; cation channel currents.

[1] V. Flockerzi, B. Nilius, in Mammalian Transient Receptor Potential (TRP) Cation Channels: Volume I (Eds.: B. Nilius, V. Flockerzi), Springer, Berlin, Heidelberg, 2014, pp. 1-12.

[2] S. Sharma, C. R. Hopkins, J. Med. Chem. 2019, 62, 7589-7602.

[3] S. Curcic, O. Tiapko, K. Groschner, Pharmacol. Ther. 2019, 200, 13-26.

[4] T. Leinders-Zufall, U. Storch, K. Bleymehl, M. Mederos y Schnitzler, J. A. Frank, D. B. Konrad, D. Trauner, T. Gudermann, F. Zufall, Cell Chem. Biol. 2018, 25, 215-223.e3.

[5] T. Leinders-Zufall, U. Storch, M. Mederos y Schnitzler, N. K. Ojha, K. Koike, T. Gudermann, F. Zufall, STAR Protocols 2021, 2, 100527.

[6] O. Tiapko, N. Shrestha, S. Lindinger, G. G. de la Cruz, A. Graziani, C. Klec, C. Butorac, W. F. Graier, H. Kubista, M. Freichel, L. Birnbaumer, C. Romanin, T. Glasnov, K. Groschner, Chem. Sci. 2019, 10, 2837-2842.

[7] J. A. Frank, M. Moroni, R. Moshourab, M. Sumser, G. R. Lewin, D. Trauner, Nat. Comm. 2015, 6, 7118.

[8] D. B. Konrad, J. A. Frank, D. Trauner, Chem. Eur. J. 2016, 22, 4364-4368.

[9] P.-Y. Lam, A. R. Thawani, E. Balderas, A. J. P. White, D. Chaudhuri, M. J. Fuchter, R. T. Peterson, J. Am. Chem. Soc. 2020, 142, 17457-17468.

[10] A. V. Zholos, in Mammalian Transient Receptor Potential (TRP) Cation Channels (Eds.: B. Nilius, V. Flockerzi), Springer Berlin Heidelberg, Berlin, Heidelberg, 2014, pp. 129-156.

[11] T. Blum, A. Moreno-Pérez, M. Pyrski, B. Bufe, A. Arifovic, P. Weissgerber, M. Freichel, F. Zufall, T. Leinders-Zufall, PNAS 2019, 116, 15236-15243.

[12] R. S. Bon, D. J. Wright, D. J. Beech, P. Sukumar, Annu. Rev Pharmacol. Toxicol. 2022, 62, 427-446.

[13] A. Minard, C. C. Bauer, D. J. Wright, H. N. Rubaiy, K. Muraki, D. J. Beech, R. S. Bon, Cells 2018, 7, 52 .

[14] H. N. Rubaiy, Br. J. Pharmacol. 2019, 176, 832-846

[15] J. M. Richter, M. Schaefer, K. Hill, Mol. Pharmacol. 2014, 86, 514-521.

[16] Y. Akbulut, H. J. Gaunt, K. Muraki, M. J. Ludlow, M. S. Amer, A. Bruns, N. S. Vasudev, L. Radtke, M. Willot, S. Hahn, T. Seitz, S. Ziegler, M. Christmann, D. J. Beech, H. Waldmann, Angew. Chem. Int. Ed. 2015, 54, 3787-3791.

[17] M. J. Ludlow, H. J. Gaunt, H. N. Rubaiy, K. E. Musialowski, N. M. Blythe, N. S. Vasudev, K. Muraki, D. J. Beech, J. Biol. Chem. 2017, 292, 723-731.

[18] H. Beckmann, J. Richter, K. Hill, N. Urban, H. Lemoine, M. Schaefer, Cell Calcium 2017, 66, 10-18.

[19] S. Just, B. L. Chenard, A. Ceci, T. Strassmaier, J. A. Chong, N. T Blair, R. J. Gallaschun, D. del Camino, S. Cantin, M. D'Amours, C. Eickmeier, C. M. Fanger, C. Hecker, D. P. Hessler, B. Hengerer, K S. Kroker, S. Malekiani, R. Mihalek, J. McLaughlin, G. Rast, J. Witek, A. Sauer, C. R. Pryce, M. M. Moran, PLOS ONE 2018, 13, e0191225.

[20] Bertrand L. Chenard, R. J. Gallaschun, Substituted Xanthines and Methods of Use Thereof, 2014, WO2014143799A3.

[21] H. N. Rubaiy, M. J. Ludlow, M. Henrot, H. J. Gaunt, K. Miteva, S. Y. Cheung, Y. Tanahashi, N. Hamzah, K. E. Musialowski, N. M. Blythe, H. L. Appleby, M. A. Bailey, L. McKeown, R. Taylor, R. Foster, H. Waldmann, P. Nussbaumer, M. Christmann, R. S. Bon, K. Muraki, D. J. Beech, J. Biol. Chem. 2017, 292, 8158-8173. 
[22] A. Minard, C. C. Bauer, E. Chuntharpursat-Bon, I. B. Pickles, D. J. Wright, M. J. Ludlow, M. P. Burnham, S. L. Warriner, D. J. Beech, K. Muraki, R. S. Bon, Br. J. Pharmacol. 2019, 176, 3924-3938.

[23] D. J. Wright, K. J. Simmons, R. M. Johnson, D. J. Beech, S. P. Muench, R. S. Bon, Commun. Biol. 2020, 3, 1-11.

[24] C. C. Bauer, A. Minard, I. B. Pickles, K. J. Simmons, E. Chuntharpursat-Bon, M. P. Burnham, N. Kapur, D. J. Beech, S. P. Muench, M. H. Wright, S. L. Warriner, R. S. Bon, RSC Chem. Biol. 2020, $1,436-448$

[25] V. A. Gutzeit, A. Acosta-Ruiz, H. Munguba, S. Häfner, A. LandraWillm, B. Mathes, J. Mony, D. Yarotski, K. Börjesson, C. Liston, G. Sandoz, J. Levitz, J. Broichhagen, Cell Chem. Biol. 2021, 28, 1648-1663.e16.

[26] M. Borowiak, W. Nahaboo, M. Reynders, K. Nekolla, P. Jalinot, J. Hasserodt, M. Rehberg, M. Delattre, S. Zahler, A. Vollmar, D. Trauner, O. Thorn-Seshold, Cell 2015, 162, 403-411.

[27] O. Thorn-Seshold, J. Meiring, ChemRxiv 2021, DOI 10.26434/chemrxiv.14424176.v1.

[28] J. Broichhagen, J. A. Frank, D. Trauner, Acc. Chem. Res. 2015, 48, 1947-1960.

[29] M. J. Fuchter, J. Med. Chem. 2020, 63, 11436-11447.

[30] X. Gómez-Santacana, S. Pittolo, X. Rovira, M. Lopez, C. Zussy, J. A. R. Dalton, A. Faucherre, C. Jopling, J.-P. Pin, F. Ciruela, C. Goudet, J. Giraldo, P. Gorostiza, A. Llebaria, ACS Cent. Sci. 2017, 3, 81-91. 